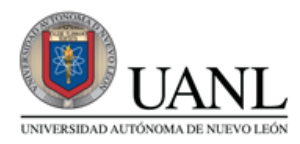

FACPYA
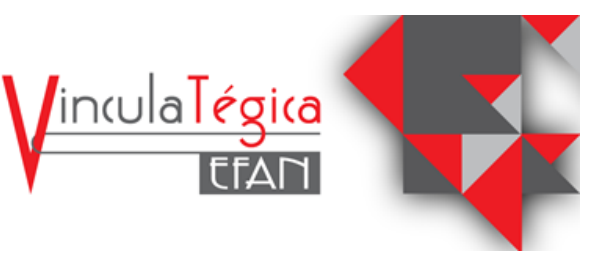

\title{
Lienzo para construir el perfil de un consumidor para un emprendimiento sostenible
}

\author{
Aniela García Antonio ${ }^{1}$, Ana Laura Luna Jiménez ${ }^{2}$ y Erika Guadalupe Ceballos Falcón ${ }^{3}$ \\ ${ }^{1}$ Universidad Juárez Autónoma de Tabasco División Académica Multidisciplinaria de los Ríos, \\ aniela.garcia@ujat.mx, C.P.86901, Tenosique Tabasco, México, Tel. (993) 3581500 Ext.6800. \\ Información del artículo revisado por pares \\ Fecha de aceptación: junio-2021 \\ Fecha de publicación en línea: diciembre-2021 \\ DOI: https://doi.org/10.29105/vtga7.2-36
}

\section{Resumen}

2021 puede ser un año que fortalecerá el emprendimiento social. Con los sucesos actuales, la tendencia mundial es hacia ver la salud como un nuevo lujo, encontrar modelos de negocio con impacto, hacer más con menos, pero sin comprometer al medio ambiente actual y futuro; y ser consumidores conscientes adquiriendo con responsabilidad (The Economist, 2020; Euromonitor International, 2019). Perfilar al cliente ideal para un nuevo producto, servicio o emprendimiento es un proceso que contiene elementos fundamentales para conocerle y formas estratégicas alrededor del mismo. Una herramienta útil para ello es el buyer persona (Zambito, 2010), sin embargo, en un estudio previo se observó que de diez lienzos analizados, ninguno cubre las necesidades de información para entender a un cliente responsable y consciente, el cual es el target de los emprendimientos sociales principalmente. Se propone un lienzo de buyer persona construido a partir de una investigación cualitativa y bibliográfica, haciendo entrevistas a emprendedores iniciales buscando resolver un problema social, así como prototipado y pivoteo del lienzo. Con ello se propone un canvas de
2021 can be a year that will strengthen social entrepreneurship. With current events, the world trend is towards seeing health as a new luxury, finding business models with impact, doing more with less but without compromising the current and future environment; and be conscientious consumers by purchasing responsibly (The Economist, 2020; Euromonitor International, 2019). Profiling the ideal client for a new product, service or venture is a process that contains fundamental elements to get to know them and strategic ways around it. A useful tool for this is the buyer persona (Zambito, 2010), however, in a previous study it was observed that of ten analyzed canvases, none covers the information needs to understand a responsible and conscientious client, which is the target of social enterprises mainly. A buyer persona canvas is proposed, built from qualitative and bibliographic research, interviewing initial entrepreneurs seeking to solve a social problem, as well as prototyping and pivoting the canvas. With this, a buyer persona canvas is proposed to define the profile of a customer of products, or projects with social impact. This material can serve in the classroom as support for entrepreneurship education, while for the development of social entrepreneurship, it could serve as an additional 
buyer persona para definir el perfil de un cliente de productos, o proyectos con impacto social. Este material puede servir en el aula como apoyo para la educación en emprendimiento, mientras que, para el desarrollo de emprendimiento social, podría servir como herramienta adicional.

$\begin{array}{llll}\text { Palabras clave: } & \text { Consumidor, } & \text { Keywords: Consumer, Sustainability, } \\ \text { Sostenibilidad, } & \text { Emprendimiento, } & \text { Entrepreneurship, Marketing. } \\ \text { Mercadotecnia. } & & \text { JEL: M39. }\end{array}$

\section{INTRODUCCIÓN}

De acuerdo con Kotler y Armstrong (2017), las empresas no cuentan con mecanismos para captar la totalidad de la población como sus clientes; y quizás, aunque lo tuvieran, no a todos les interesaría el mismo tipo de producto o servicio que se ofrece. De ahí que la segmentación de mercado sea una herramienta fundamental cuando se busca delinear al posible comprador. Con el paso de los años, las técnicas para definir al cliente ideal han evolucionado.

Principalmente podemos encontrar dos: la segmentación de mercado, que eventualmente se ha llevado a nichos de mercado; y la construcción de un buyer persona (Zambito, 2010) o un user persona creado por Alan Cooper (UX Poland, 2017). Debido a la COVID-19, las empresas están priorizando el entendimiento de los clientes ya que sus actitudes de compra y comportamiento mismo ha cambiado a propósito del contexto actual (KPMG, 2021).

Este proyecto es la continuación de un proceso de investigación para fortalecer la construcción del cliente ideal en los emprendimientos, ya que en el desarrollo de un modelo de negocio, es necesario entender quién es el cliente, sin embargo, al hacer una evaluación de los buyer persona, la cual, es una herramienta que funciona para determinar las características principalmente de quien consideramos sería el consumidor principal, se pudo observar que solamente dos tienen los elementos necesarios y adecuados de acuerdo con su creador, Alan Cooper (UX Poland, 2017). Sin embargo, utilizarlo para el tool.

desarrollo de modelos de negocio de base sostenible no sería lo más adecuado puesto que en los lienzos no se aclaran algunos puntos que un consumidor responsable observa como su propósito y sus intereses y pensamientos para con el mundo.

De esta manera, presentamos una propuesta de lienzo de buyer persona la cual funcionará principalmente en el proceso de modelos de negocio sustentables, o que inclusive funcionan para proyectos de economía social y solidaria. Realizamos este proyecto para definir un buyer persona delineado para un emprendimiento que sea sostenible lo cual requiere de tener claros algunos puntos, como entender el propósito de vida del cliente, las intenciones de compra y los motivos que tiene día a día.

\section{MARCO TEÓRICO}

Sea en marketing, en la administración de productos $\mathrm{o}$ en el arranque de un emprendimiento, la comprensión de un cliente es crucial si se busca que la empresa genere mayores beneficios para si mismo y para su comunidad. Es por ello por lo que comprender quién es un cliente para un proyecto de base sustentable no se había tornado tan relevante como en la actualidad.

\subsection{El cliente}

Es necesario comenzar por comprender quién es un cliente. De acuerdo con López Salas (2020) un cliente es "la persona física o jurídica que compra en tiendas o establecimientos, o utiliza con asiduidad los servicios de profesionales o empresas a cambio de una transacción monetaria". 
Martínez (2014), menciona que un cliente primero que todo es una persona, y es la razón del éxito o fracaso de una empresa. Asimismo, dice que pueden ser actuales, es decir, quienes actualmente con sus compras generan utilidades a la empresa; y potenciales, quienes, aunque no han comprado, tienen el interés y la autoridad para hacerlo en algún momento.

Finalmente, para Carvajal et. al (2019), un cliente es una persona que adquiere algo de manera habitual; es decir, que ya tiene conexión con el producto, le ha funcionado y por lo tanto decide continuar comprándolo. Es prioridad comprender que los clientes son personas que toman decisiones que eventualmente se pueden convertir en un producto o servicio adquirido, no al revés. $\mathrm{La}$ miopía del marketing (Kotler y Armstrong, 2017), que es poner por encima de las necesidades del cliente al producto o servicio en venta, puede generar un problema en el desempeño de las utilidades empresariales. Si bien es necesaria la comprensión de la propuesta de valor (Osterwalder et. al, 2014), ya que de esta manera se tiene un contexto claro de lo que se ofrece en conjunto con lo que el cliente desearía y está perdiendo por no tenerlo en la actualidad; es también requisito delinear al posible cliente con el fin de tener más información para tomar decisiones de marketing, de ventas y financieras.

\subsection{Métodos para conocer al cliente}

En la actualidad, para definir al cliente ideal, se utiliza la investigación de mercados (Malhotra, 2004), sea cualitativa o cuantitativa, en donde, a través de diferentes herramientas se llega a una conclusión con la que se identifica a esa persona que quizás, adquiera el producto o servicio. La investigación de mercado tiene ventajas principalmente estadísticas, no obstante, los tiempos para su realización son mayores cuando hablamos de un desarrollo de modelo de negocio o de tener un primer panorama de dicho cliente. Para la construcción de un modelo de negocio es primario conocer al cliente con quien se validará la propuesta de valor, para ello en el Bussines Model Canvas (Osterwalder y Pigneur, 2010) y Lean Canvas (2014), existe un espacio que, si bien es pequeño, es aquel donde se fincan los puntos importantes de este grupo de personas que eventualmente pudieran comprar el producto.

Adicional a ello, Osterwalder, et.al (2014), desarrollaron el Lienzo de la Propuesta de Valor, en el cual, se genera un mapa de los trabajos del cliente, las ganancias $y$ frustraciones de la persona al querer resolver un problema, y con ello, encontrar soluciones a dichas frustraciones que representen al final un producto o servicio y que este encaje con lo que el cliente busca resolver. Es decir, el lienzo es un espacio donde depositar las ideas, pensamientos, observaciones e ideas que se tienen alrededor del comportamiento de una persona para con ello construir una mejor idea de negocio.

Por otra parte, en el desarrollo de productos desde la perspectiva del marketing, se ocupa la segmentación de mercados como herramienta para definir con características como su estilo de vida, motivaciones, demografía y comportamientos, a la persona que podría adquirir el producto o servicio, pero también para tener un marco de información para la elaboración del plan de marketing y el desarrollo de herramientas como la publicidad, relaciones públicas o ventas por mencionar algunos. La segmentación de mercados de acuerdo con Charles Lamb (2010) es la división de grupos de un mercado que tienen características similares.

Asimismo, de acuerdo con Mestre, Villar y Guzmán (2014) la segmentación de mercado es la división del mercado en subgrupos homogéneos con el fin de llevar a cabo una estrategia comercial diferenciada. Bajo este precepto se podría considerar que el ejercicio de dividir a las personas a nivel comercial es para darle foco a un proyecto de comercialización como tal, sin embargo, el proceso mismo de la indagación en el cliente es la fuente de información para la definición de un proyecto nuevo, sea un emprendimiento, nuevo producto a lanzar o estrategia.

\subsection{Buyer Persona}

Existen diversos conceptos alrededor de lo que un buyer persona es, pese a ello, desde la perspectiva conceptual y de su ejecución, estos coinciden en tres aspectos primordiales: a) es 
un depósito de información, b) funciona para conocer al cliente ideal; y c) es una radiografía construida con lo que se conoce del consumidor que además ayuda a tomar decisiones. Como Hubspot (Sordo, 2019) menciona, es "una representación semi ficticia de tus clientes ideales".

De acuerdo con Alan Cooper, funciona para tener claridad en el proceder de un usuario en el momento de tomar una decisión sea de compra o de rechazo de la misma (UX Poland, 2017). Adicionalmente a ello, Alguacil et.al (2019) opina que es una descripción sociodemográfica para el perfil del comprador y explicado a detalle en sus características. Nielsen Norman Group (2018) por su parte, consideran que el buyer persona es una representación de un nicho de mercado que con comportamientos y metas parecidas con respecto a los productos y servicios de una compañía. Finalmente, hablando de cómo construir un buyer persona, Revella (2015), considera que entrevistando compradores que pasaron por su proceso de compra, incluyendo momentos de frustración de la misma, se podría encontrar más información, puesto que esta ya se encontraría interiorizada.

El buyer persona, desde la revisión bibliográfica realizada, es una herramienta primariamente utilizada en el desarrollo de emprendimientos, y desde la consultoría empresarial, por lo que encontrar algún lienzo o método para elaborarle, supone facilidad a través de un blog o página web que por medio de un libro o artículo científico. Una posibilidad es que con la investigación de mercados se crean perfiles de clientes. Autores como Shrun, Mcarty, Lowrey (1995), Elhoushy y Lanzini (2020) y Han y Stoel (2016), han hecho estudios alrededor de las características de un consumidor verde o consumidor responsable, así como el comportamiento de ellos, dando información relevante y que enriquece el conocimiento de este nicho de mercado, sin embargo, el propósito de un lienzo de buyer persona es dar las primeras directrices de dicho cliente, y que quienes ocupen esta herramienta tengan un panorama de lo que con continuidad, necesiten saber al momento de desarrollar un proyecto de emprendimiento. En la búsqueda de conocer a un tipo de cliente, la investigación exploratoria o el uso de herramientas como la observación, entrevistas o sondeos ayudan a nutrir y a construir un modelo específico de consumidor que va de acuerdo al contexto donde se busca desarrollar el proyecto emprendedor. Las fuentes secundarias se convierten en un soporte que fortalezca lo realizado a través del trabajo de campo.

\subsection{Consumo responsable}

El consumo responsable, si bien no es una actividad nueva, ha tomado fuerza con el paso de los años movido básicamente por dos aspectos. Por una parte, se encuentra la Agenda 2030 de la Organización de las Naciones Unidas (2015) en la cual, a través de 17 objetivos buscan que ninguna persona se quede rezagada en ningún aspecto, sea alimenticio, calidad de vida o empleo por mencionar algunos. $\mathrm{Y}$ es aquí donde se presenta el Objetivo 12 "Producción y consumo responsables", donde, de acuerdo con la ONU (2020) buscan "comprender y valorar los límites hasta los que podemos presionar a la naturaleza antes de que su impacto sea negativo. Dichos límites se deben reflejar en nuestros patrones de consumo y producción".

Aunado a ello se encuentra la conciencia colectiva de las nuevas generaciones, en donde representantes como Greta Thumberg (Tedx Talks, 2018) apela a expresar su inconformidad por los mecanismos actuales desde diversas industrias y procesos que no permiten que disminuyan las amenazas de un inminente problema climático. Con base en las Tendencias de Consumo Global realizadas por Euromonitor (2021) y en las predicciones 2021 de The Economist (2020), el consumidor se va a convertir en una persona con mayor conciencia en lo que adquiere y por qué lo hace. Asimismo, se convertirá en un tipo de cliente frugal, interesado en adquirir artículos de segunda mano como una representación de su interés por disminuir la sobre producción de artículos como la moda rápida; adicionando a ello, su mayor participación en las compras de productos que en su proceso no le hayan afectado más al medio ambiente. 
Entender entonces al consumidor y tener claridad en el tipo de persona que es, sus motivaciones, sus dolores y pensamientos frente al consumo responsable serán un patrón de información crucial en las nuevas empresas y proyectos futuros.

\subsection{Emprendimiento social}

El cliente es el foco y centro de los nuevos proyectos y las respuestas a las nuevas disyuntivas para solucionar problemas, toda vez que de ahí emanan las nuevas oportunidades para la innovación en los productos, el relanzamiento de empresas o la solución de problemas mundiales. En este último se finca el concepto de la economía y los emprendimientos sociales. De acuerdo con la Guía sobre la Economía Social y Solidaria (D.C.S., 2010), las empresas de la economía social son "creada con la meta explícita de beneficiar a la comunidad, iniciada por un colectivo de ciudadanos, y en la que el interés material de los inversores de capital está sujeto a límites [...] además, ponen especial valor en su autonomía y en el riesgo económico derivado de su continua actividad socioeconómica".

Por su parte Guzmán y Trujillo (2008), mencionan que no hay conceptos unificados de lo que es el emprendimiento social, sin embargo, se congenia en que son entes empresariales que buscan resolver problemas sociales como la contaminación, el analfabetismo o la drogadicción. Por su parte, en el Glosario de Inversión de Impacto (Ashoka, Aspen Network of Development Entrepreneurs (ANDE), Factual, \& Promotora Social México, 2014), menciona que una empresa social es una "organización dedicada a actividades industriales, mercantiles o de prestación de servicios que busca tener un impacto social o ambiental positivo y medible. Dicho impacto debe ser de forma sostenible, es decir, que genere sus propios recursos para seguir desarrollando sus actividades."

Las empresas sociales están teniendo un empuje mayor conforme el tiempo y el conocimiento avanza. De acuerdo con Juan del Cerro (2016), pionero en el ecosistema del emprendimiento social en México, hay diferencias entre un emprendimiento social, la filantropía y las empresas socialmente responsables. Esto también lo ha llevado a entender que hay algunas diferencias en como construirlas y fortalecerlas con las herramientas que actualmente se ocupan para modelar negocios que no ven al impacto en la sostenibilidad como prioridad. Para este caso, consideramos al cliente ideal, es decir, el buyer persona (Zambito, 2012). Al haber realizado un análisis previo de los lienzos actuales, se ha analizado que los elementos cruciales como el impacto y el propósito no son evaluados en los formatos analizados.

Construir pues un lienzo de buyer persona que tenga un enfoque hacia el conocimiento de un cliente responsable o consciente es un mapa que hasta el día de hoy en que se redacta el presente proyecto, no se encuentra en bibliografías impresas o digitales, por ello, y porque el emprendimiento social, los proyectos sustentables y de impacto continúan creciendo, se considera al cliente un elemento fundamental, y por lo tanto, le da valía a todo esfuerzo alrededor de encontrar mecanismos que abonen a su conocimiento.

\section{MÉTODO}

Para la realización del lienzo, se realizó una investigación previa con la cual se desarrolló un estudio de los diez lienzos de buyer persona más ocupados, con lo cual se conducirá una parte de la construcción del lienzo de buyer persona.

Seguidamente, se realizó una investigación cualitativa de alcance transversal a través de la herramienta de entrevista a profundidad (Malhotra, 2004), a tres emprendedoras con proyectos que tienen perspectiva sustentable. Con el prototipo del lienzo, se realizó la validación con el grupo de emprendedoras, y con cuatro expertas de la educación en emprendimiento de la Universidad Juárez Autónoma de Tabasco. Finalmente, se realizó el pivoteo del lienzo, a través de un taller en línea con alumnos de la materia de desarrollo sustentable de la carrera de Administración en la División Académica Multidisciplinaria de los Ríos de la UJAT. De esta forma, se pudo obtener un lienzo validado utilizando las reglas del Lean Startup (Rice, 2014). Se considera entonces que el objetivo general de 
este proyecto fue la elaboración de una propuesta de lienzo de buyer persona para definir al cliente ideal de un emprendimiento sustentable. Es importante mencionar que debido a las condiciones de confinamiento por la COVID-19, el proceso de investigación, validación y pivoteo, requirió ser ejecutado a través de medios electrónicos. Sin embargo, se considera haber encontrado insights valiosos que permitieron su construcción y su revisión posterior.

\section{RESULTADOS}

Como se mencionó, para la realización de este lienzo, se realizaron tres entrevistas a emprendedoras de tres industrias distintas: tienda en línea de ropa de segunda mano; café de maíz artesanal y macetas hechas con fibra de coco. Se aplicó una guía de entrevista en la cual se buscó la información acerca de su modelo de negocio, el conocimiento actual que tienen de su cliente, las generalidades en torno al desarrollo sustentable y la economía circular; y acerca de estrategias de marketing y sustentabilidad. Se les preguntó también por su conocimiento de la metodología de buyer persona, esto como un indicador del panorama de su uso para el desarrollo de proyectos sustentables.

Con respecto a su modelo de negocio cada proyecto mencionó resolver un tema fincado en mejorar el bienestar de las personas sin vulnerar al medio ambiente. Por ejemplo la emprendedora Elena Tepal (ropa de segunda mano) dijo que se trata de "vender lo que no usas y comprar lo que ya no usan"; Nínive Cristina Pérez (macetas hechas con fibra de coco) mencionó que su modelo de negocio se basa en resolver el problema de "reducir el plástico en macetas y evitar la quema de cascaras de coco" principalmente; mientras que Iridian Delgado emprendedora con el producto de café de maíz, mencionó que este negocio se dedica al "desarrollo de una bebida a base de maíz con sabor a café [con] granos de maíz totalmente naturales".

Con base en el conocimiento que tienen actualmente de su cliente, las emprendedoras mencionaron que "en su mayoría mujeres, muy amantes a la naturaleza, les gustan mucho los colores vivos o verdes, siempre tiene en Facebook o en WhatsApp fotos de plantas o paisajes, comparten contenido del cuidado del medio ambiente. A muchas o muchos les gusta leer, el café, los viajes y eso me lo dicen sus estados en Facebook o WhatsApp"; para Elena Tepal son "principalmente mujeres que no tienen mucho interés por las últimas tendencias de moda. El consumo de la ropa de segunda mano ha crecido y seguirá creciendo por el hecho de que responde a la tendencia de la sostenibilidad ecológica"; finalmente para Iridian Delgado, su cliente ha evolucionado ya que como menciona la emprendedora "nuestro consumidor meta eran personas con enfermedades que les dificultara o les hiciera nula la posibilidad de consumir la cafeína, brindando una opción libre de cafeína. Posteriormente a una investigación, nuestro mercado se dirige a las mujeres debido a problemas de fibrosis, menopausia o SPMP. Sin embargo, el giro viene cuando a la venta nuestros clientes han sido personas sin especificaciones de salud que han adquirido el producto por el interés de probar algo nuevo."

Para responder a las generalidades en torno al desarrollo sustentable y la economía circular, se les preguntó a las emprendedoras su opinión con respecto a la economía circular y la sustentabilidad; para ello, sus respuestas, aunque variadas, pudieron converger en el propósito latente de hacer de la sustentabilidad y la economía circular reglas básicas para el desarrollo de nuevos productos $\mathrm{o}$ emprendimientos mismos. Para la emprendedora del café de maíz, la economía circular y el desarrollo sustentable "ofrece una opción importantísima en la actualidad y da respuesta y dirección a las empresas y futuras empresas hoy en día, sobre la posibilidad de vender sin generar un impacto ambiental"; por su parte, Nínive Cristina Pérez mencionó que "es lo que muchas empresas deben tomar en cuenta y la gente como consumidores también , por qué nuestro futuro no es desechable y los recursos naturales algún día pueden agotarse, ya no habrá para producir y lo peor es que morirnos junto con ellos"; finalmente, para Elena Tepal mencionó que hablando de la economía circular "la idea es muy buena, quiere imitar el proceso natural donde nada se 
desperdicia que es cien por ciento circular, así podemos darle una nueva vida o propósito a cualquier producto".

Con respecto a las generalidades acerca de las estrategias de marketing y la sustentabilidad, las entrevistadas mencionaron lo siguiente: para Nínive Cristina Pérez las estrategias de marketing que consideran sustentables son "WhatsApp, Facebook [e] Instagram, es poco pero el día que hicimos la página de Facebook juntamos 100 seguidores"; con respecto a ello Elena Tepal mencionó, ella menciona que "el inbound marketing"; mientras que para Iridian Delgado menciona que el "marketing por redes sociales" y marketing de contenido. Y esto considero que se debe a la famosa era digital ya que en la actualidad es muy poco probable que no se cuente con algún dispositivo mayormente un teléfono celular, desde la clase baja hasta el alta. Sin embargo, considero que el marketing que nunca dejará de funcionar es aquel que surge de las recomendaciones de persona a persona, basado en la experiencia."

Finalmente, cuando se les preguntó si conocen el buyer persona mencionaron lo siguiente: "En una ocasión tuve la oportunidad de leer vagamente sobre ello, es un concepto funcional para recrear el cliente ideal con el cual las empresas puedes hacer un mejor desarrollo de producto", mencionó Iridian Delgado; "Claro, es la información precisa del cliente ideal" mencionó Elena Tepal; y finalmente Nínive Cristina Pérez mencionó que "no había escuchado" hablar de esta herramienta.

Con esta información, se ha podido tener un análisis de como es actualmente la operación de dichos proyectos y como un elemento como un buyer persona sería importante para tener claridad de quien es el cliente, sobre todo, por la manera en la que este producto o servicio será ofrecido.

Algunos temas para revisar están en el beneficio que las emprendedoras ven en el desarrollo sustentable y en la necesidad de tener una herramienta que les ayude a tener gráficamente un panorama de su cliente. Para ello, se propone un lienzo con el cual puedan modelar su proyecto que bien puede servir como un repositorio de información y que interactivamente puedan ir evolucionando. Al preguntárseles si ven beneficios en la utilización de un lienzo para determinar a su cliente ideal, las entrevistadas mencionaron que sí. Con dicha respuesta se acepta entonces la necesidad de la construcción de dicho elemento.

Se determinó realizar un lienzo basándose en las reglas de Tony Zambito (2012) e incluyendo preguntas de elaboración propia que reflejen el objetivo de la perspectiva de consumo responsable.

Los componentes clave que se plasman en el lienzo del consumidor responsable ideal que se presenta, contiene como base las cinco reglas que Zambito (2012) menciona para su elaboración. Estas reglas son:

1. Investigación de los insights del comprador/consumidor: Para responder a esta regla se consideraron los bloques tres y cinco

2. Descripción del arquetipo del comprador/consumidor: Esto basado en el concepto de los arquetipos (Jung, 2014), lo cual le da un panorama fincado desde una perspectiva actitudinal decantado en los bloques siete y ocho (¿a qué arquetipo pertenece?)

3. Escenarios del comprador/consumidor ideal: para ello se requiere información de la persona desde el perfil sociodemográfico, así como sus motivaciones y creencias, lo cual abona al entendimiento del comportamiento del consumidor. Para ello, se requiere del llenado de los bloques cuatro y nueve

4. Modelos mentales del comprador/consumidor: en el se informará acerca de las ideas, actitudes y creencias que tiene la persona a analizar. Para ello se ocuparán los bloques tres y cinco.

5. Estrategia del comprador/consumidor: Para entender el proceso de compra del consumidor, se rellenarán los bloques seis y diez.

Adicionalmente a ello, se le adiciona los 
bloques dos y diez. El primero se conduce a conocer los puntos de dolor, el cual es un recurso tomado del Lienzo de la Propuesta de Valor (Osterwalder et. al, 2014)), ya que a través de este apartado se dan a conocer las molestias y sinsabores de los consumidores, es decir, plasman sus frustraciones, de las cuales podremos inclusive encontrar el Job to be Done (Christensen, et. al, 2007). Adicionalmente, se ocupará el bloque 10 como apartado para tener una aproximación de cómo el proyecto pensado podría hacerle la vida mejor al consumidor; pero, además, cómo esto tendría impacto con las creencias y motivos de dicha persona. Este bloque es de elaboración propia, basado en la experiencia recabada a través de la colaboración en el desarrollo de proyectos de emprendimiento universitarios.

Utilizando la metodología de aprendizaje validado de Lean Startup (Rice, 2012), se llevaron a cabo validaciones del prototipo. Primero que todo se hizo una valoración entre expertos de formación de emprendedores y recomendaron que se le diera un número a cada bloque para conocer el inicio y fin del ejercicio; además comentaron que se clarificara el proceso con el tema del consumidor responsable; y con ello se modificó la búsqueda de información para el bloque tres, donde en lugar de preguntar la opinión de las personas por el consumo responsable, se le cuestiona al emprendedor o quien use la herramienta para que mencione las actitudes que considera que el cliente ideal tiene frente al consumo responsable.

Después de los cambios hechos con base en las recomendaciones al prototipo, se hizo una segunda validación con 13 alumnos de la materia "Desarrollo Sustentable" en la cual, después de haberlo ejecutado, mencionaron que fue un elemento sencillo de usar y consideraron que sí es una herramienta útil para conocer a su comprador ideal. Es importante mencionar que para poder rellenar el bloque ocho, se les entregó la información pertinente a los Arquetipos de acuerdo con Carl Gustav Jung (2014).

El resultado se presenta en la figura 1. En la cual, como se aprecia, aparecen diez bloques en donde mapear la información recabada de su cliente o consumidor ideal. Es importante precisar, que para este proceso, cliente o consumidor lo consideraremos de la misma manera. 
Figura 1. Lienzo de buyer persona para proyectos sustentables.

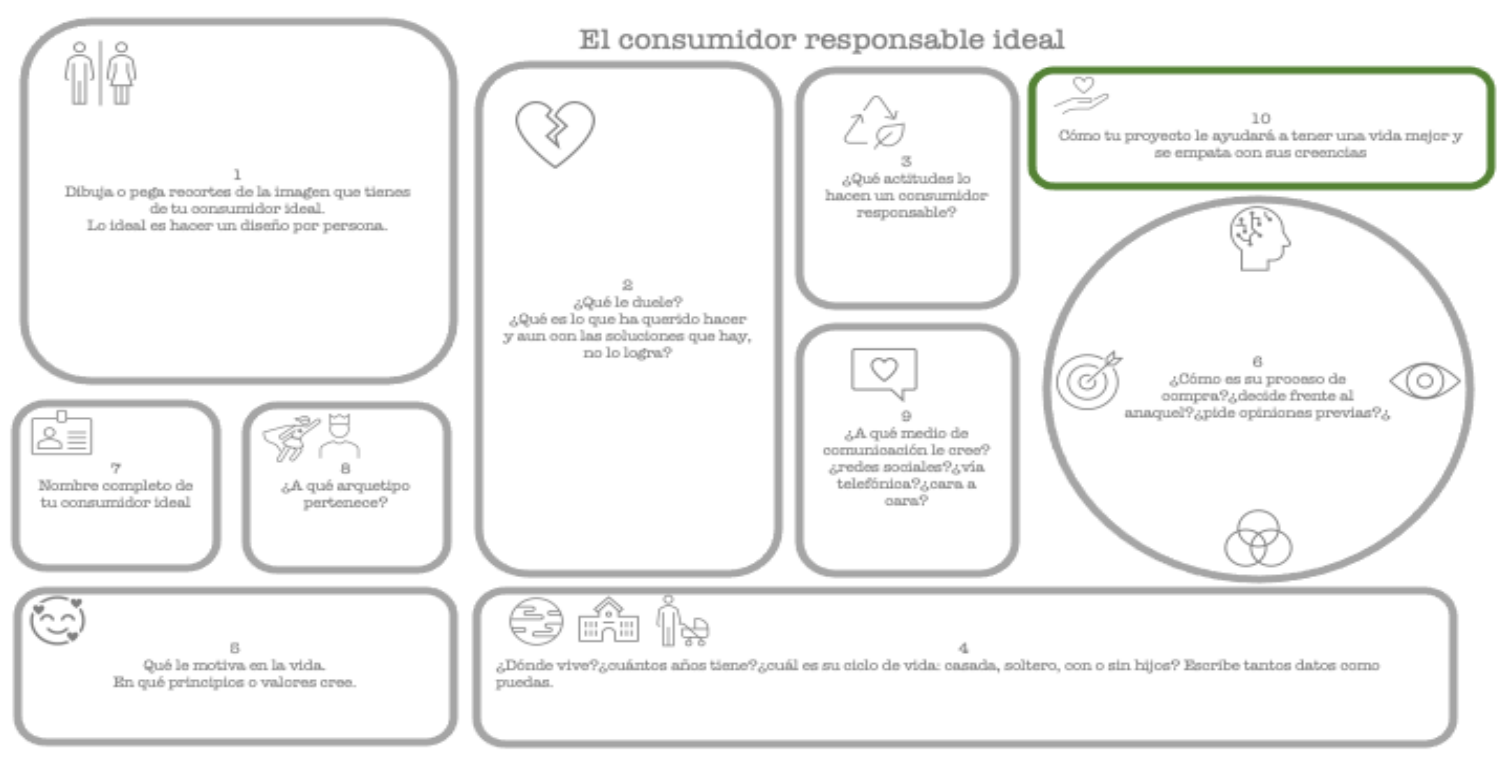

\section{CONCLUSIONES}

Centrarse en el cliente es un factor fundamental en el desarrollo de emprendimientos con propósito y que sean sostenibles en el tiempo. Asimismo, tener una idea de quién sería la persona que comprara el producto o lo usara da más confianza a quien está determinando el modelo de negocio o el producto a lanzar.

Con el desarrollo de este lienzo para la construcción de un buyer persona con especificación para proyectos o emprendimientos sostenibles, se abre la posibilidad de que se finquen mejores resultados en la comprensión de un tipo de consumidor que además de pensar en resolver un problema, también busca hacerlo con integridad, sin generar mayor impacto en el medio ambiente o en su comunidad.

Es recomendable tener la información necesaria del cliente debido a que es la llave al desarrollo de productos y servicios apegados a lo que estos requieres. Hoy por hoy, el impacto que una empresa genera con lo que ofrece es un parámetro necesario para demostrar en los proyectos, ya que el nicho del consumo responsable busca empresas comprometidas con el menor daño al mundo posible, de ahí que sea tan necesario el conocimiento de las personas que toman la decisión de adquirir sus bienes o servicios con un propósito claro.
Este lienzo es la decantación de estudio, pivoteo y análisis de distintas propuestas predeterminadas que actualmente se ofrecen en espacios digitales y en el cual, hasta que se culmina la escritura de este artículo y a nivel de estudios de investigación, no se ha encontrado alguna propuesta similar. Se han encontrado estudios en el cual se determinan características de un consumidor responsable, sin embargo, en el ejercicio de determinar a un buyer persona, es importante que quien esté desarrollándolo utilice su experiencia así como sus sentidos para poder entender, empatizar e interiorizar las condiciones que tendría dicho cliente ideal. Es por ello por lo que un lienzo en el cual se pueda iterar la información sería de utilidad en el ejercicio de construcción de este consumidor. Asimismo, consideramos esto como una colaboración en la cual se aporta un camino hacia un análisis detallado, sencillo y estratégico de lo que actualmente conocemos como el cliente ideal.

Se abre la posibilidad para continuar con investigaciones alrededor del consumidor responsable, como su perfil y los problemas que actualmente quisieran resolver lo cual pudiera derivar en nuevos productos, servicios y emprendimientos mismos.

Asimismo, se espera continuar con la validación del lienzo en talleres y en aulas de clase para fortalecer su uso 


\section{REFERENCIAS}

Alguacil, M., Crespo-Hervás, J., \& Pérez-Campos, C. (2019). Análisis sociodemográfico de la percepción de marca en un servicio deportivo público: del target al buyer persona (Sociodemographic analysis of brand perception in a public sports service: from target to person buyer). Retos, 37, 139-146. https://doi.org/10.47197/retos.v37i37.71707

Ashoka, Aspen Network of Development Entrepreneurs (ANDE), Factual, \& Promotora Social México. (2014, 9 mayo). Glosario de Inversión de Impacto. Glosario de Inversion de Impacto. https://glosarioinversionimpacto.wordpress.com/author/glosarioinversionimpacto/

Cerro, D. J. (2016). ¿Qué es el Emprendimiento Social? (1.a ed.). NEISA.

Carvajal, L. G., Ormeño, J., \& Sanz, L. (2019). Atención al Cliente. Editex. https://books.google.com.mx/books?id=MYmXDwAAQBAJ\&printsec=frontcover\&dq=Carv ajal,+Orme \%C3\%B1o+y+Sanz+cliente\&hl=es\&sa=X\&ved=2ahUKEwj_iOOD5srwAhUSM awKHS24DvIQ6AEwAHoECAMQAg\#v=onepage $\& q=$ Carvajal $\% 2 \mathrm{C} \% 200 \mathrm{rme} \% \mathrm{C} 3 \% \mathrm{~B} 1 \mathrm{o} \%$ $20 \mathrm{y} \% 20 \mathrm{Sanz} \% 20$ cliente $\& \mathrm{f}=$ false

Christensen, C., Anthony, S. D., Berstell, G., \& Nitterhouse, D. (2007, 1 abril). Finding the Right Job For Your Product. MIT Sloan Management Review. https://sloanreview.mit.edu/article/finding-the-right-job-for-your-product/

D.C.S. (2010). Guía sobre la Economía Social y Solidaria (1 a ed.). Confederación Sindical de CCOO.

Elhoushy, S.\& Lanzini, P. (2020): Factors Affecting Sustainable Consumer Behavior in the MENA Region: A Systematic Review, Journal of International Consumer Marketing, DOI: $10.1080 / 08961530.2020 .1781735$

Esquivel, V. (2021). Panorama 2021: Cuatro prioridades en una nueva realidad. KPMG.

Euromonitor Internacional. (2019). Understanding the Socioeconomic Drivers of Megatrends.

https://go.euromonitor.com/white-paper-megatrends-2019-understanding-the-socioeconomicdrivers-of-megatrends.html

Euromonitor International. (2021, enero). Top 10 Global Consumer Trends. https://go.euromonitor.com/white-paper-EC-2021-Top-10-Global-Consumer-Trends.html

Godin, S. (2019). Esto es marketing. Alianza Editorial.

Han, T.\& Stoel, L. (2016): Explaining Socially Responsible Consumer Behavior: A Meta-Analytic Review of Theory of Planned Behavior, Journal of International Consumer Marketing, DOI: $10.1080 / 08961530.2016 .1251870$

Hubspot. (2020). Generador de buyer personas. https://www.hubspot.es/make-my-persona

Jung, C. G. (2014). Arquetipos e inconsciente colectivo. Paidos México.

Kotler, P., Kartajaya, H., \& Setiawan, I. (2021). Marketing 5.0. Wiley.

Kotler, P., \& Armstrong, G. (2017). Marketing (16.a ed.). Pearson Educación.

Lamb, C. W., Hair, J. F., \& McDaniel, C. (2010). Marketing (11.a ed.). South-Western Pub.

López Salas, S. (2020). Atención al cliente, consumidor y usuario. Paraninfo

Malhotra, N. (2008). Investigacion De Mercados (5.a ed.). Pearson Educación.

Maurya, A. (2012). Running Lean. Van Duuren Media.

Martínez, P. M. (2014). Atención al cliente (1a ed., 1a imp. ed.). Paraninfo. https://books.google.com.mx/books?id=WWWgBwAAQBAJ\&printsec=frontcover\&dq=palo mo+cliente+2014\&hl=es\&sa=X\&ved=2ahUKEwj_uMG858rwAhUFIqwKHUTADd0Q6AE wAHoECAIQAg\#v=onepage $\& \mathrm{q}=$ palomo\%20cliente $\% 202014 \& \mathrm{f}=$ false

Mestre, M. S., Villar, F. J. V., \& Guzmán, A. D. C. S. (2014). Fundamentos de Mercadotecnia. Grupo Editorial Patria.

Nielsen Norman Group. (22 de febrero de 2020). Why Personas Fail [Vídeo]. YouTube. https://www.youtube.com/watch?v=fal4Wylyt3M

Osterwalder, A., \& Pigneur, Y. (2010). Business Model Generation: A Handbook for Visionaries, Game Changers, and Challengers (1st ed.). Wiley

Osterwalder, A., Pigneur, Y., Bernarda, G., Smith, A., \& Papadakos, T. (2014). Value Proposition Design. Wiley. 
Revella, A. (2015). Buyer Personas: How to Gain Insight Into Your Customer's Expectations, Align Your Marketing Strategies, and Win More Business. Wiley.

Ries, E., \& Julián, J. S. (2012). El método Lean Startup. Deusto.

Shrum, L.J; McCarty, J. \& Lowrey, T. (1995) Buyer Characteristics of the Green Consumer and Their Implications for Advertising Strategy, Journal of Advertising, 24:2, 71-82, DOI: 10.1080/00913367.1995.10673477

Tedx Talks. (2018, 12 diciembre). School strike for climate - save the world by changing the rules | $\begin{array}{llll}\text { Greta Thunberg } \quad \text { TEDídeo]. YouTube. } & \end{array}$ https://www.youtube.com/watch?v=EAmmUIEsN9A

The Economist. (2020, 16 noviembre). The World In 2021. https://www.economist.com/the-worldin-

2021?fbclid=IwAR2IkD06_ah2YDCFTmJp7XkJs3AzuFOzOIQRTa7ZS9DmQrT8vasnjSjeU $1 Y$

UX Poland. (14 noviembre de 2017). Working Backwards | Alan Cooper | UX Poland 2017 [Vídeo]. YouTube. $h t$ ttps://www.youtube.com/watch? $\mathrm{v}=\mathrm{z} 2 \mathrm{AHeZSw} 4 \mathrm{pU} \& \mathrm{t}=85 \mathrm{~s}$

Zambito, T. (19 enero de 2010). The Origin of Buyer Personas. Buyer Persona Insights. https://www.buyerpersonainsights.com/2010/01/the-origin-of-buyer-personas.html

Zambito, T. (Junio de 2012). The State of Buyer Personas 2012. http://tonyzambito.com/wpcontent/uploads/2013/05/The-State-of-Buyer-Personas-2012.pdf

Zambito, T. (2013). Buyer Persona Development [Plantilla]. http://tonyzambito.com/wpcontent/uploads/2013/09/Buyer-Persona-Development.pdf 